\title{
Experimental Evaluation of Read Performance for RFID-based Mobile Sensor Data Gathering Applications
}

\author{
lain A. Currie \\ I.A.Currie@sms.ed.ac.uk \\ Mahesh K. Marina \\ mmarina@inf.ed.ac.uk \\ School of Informatics \\ The University of Edinburgh, UK
}

\begin{abstract}
We consider RFID-based sensing applications enabled by passive or semi-passive tags and mobile devices equipped with readers. We experimentally investigate the feasibility of such RFID-based mobile sensor data gathering applications, focusing on UHF RFID devices and indoor scenarios. We examine the impact of various factors, including reader mobility, multiple closely located tags and other key related metrics. Our measurement results suggest the feasibility of using RFID for such applications.
\end{abstract}

\section{Categories and Subject Descriptors}

C.2.1 [Computer-Communication Networks]: Network Architecture and Design—wireless communication

\section{General Terms}

Experimentation, Measurement, Performance, Reliability

\section{Keywords}

Mobile sensor data gathering, Smart phones, RFID-enabled mobile devices, RFID sensors.

\section{INTRODUCTION}

Radio Frequency IDentification (RFID) [1, 2] is a wireless communication technology intended as an alternative to bar-codes for automatic identification of objects. Although not as cheap as barcodes, RFID tags offer several powerful capabilities that make them more flexible and widely applicable. Unlike bar-codes, RFID tags provide a larger set of unique IDs and allow fast identification of multiple co-located tagged objects from a distance without requiring line of sight. Moreover, RFID tags can have embedded computing capabilities, store much more additional data beyond the ID information and can also be interfaced with environmental sensors and digital data sources. The lowering costs and increasing sophistication of RFID tags coupled with the emergence of standards (e.g., EPCglobal Class 1 Gen 2 standard [3] and the associated ISO

Permission to make digital or hard copies of all or part of this work for personal or classroom use is granted without fee provided that copies are not made or distributed for profit or commercial advantage and that copies bear this notice and the full citation on the first page. To copy otherwise, or republish, to post on servers or to redistribute to lists, requires prior specific permission and/or a fee.

MUM'2008, December 3-5, 2008, Umeå, Sweden.

Copyright 2008 ACM 978-1-60558-192-7 08/12 ...\$5.00.
18000-6C) have led to significant and renewed interest in this technology with a plethora of applications in diverse domains, including supply chain, retail, transportation and healthcare for tracking, access control and wireless commerce.

We consider an important and emerging class of RFID applications - the use of RFID in sensing applications $[4,5]$. Of particular interest in this context are passive and semi-passive tags equipped with sensing capabilities as they can potentially last a very long time while still being fairly cheap. Passive sensor tags like ordinary passive tags are powered by readers, so can sense only in the presence of a reader. Semi-passive tags like passive tags depend on power from reader for communication, but use a battery for continuous sensing. Several instances of integrating sensing capabilities into passive and semi-passive RFID tags exist, both commercially and as research prototypes. Examples on the commercial side include VarioSens temperature logger tag from KSWMicrotec [6] and temperature logger UHF semi-passive tag from CAEN RFID [7]. Notable research prototypes include bacterial sensor tags from Auburn University mentioned in [4] and WISP battery-less passive tags from Intel [8].

Leveraging the emerging trends toward mobile handheld devices equipped with compact, power-efficient and low cost RFID readers, we consider a mobile sensor data gathering paradigm based on RFID technology for low cost sensing in indoor environments (including offices, homes and hotspots). It essentially involves gathering data from densely deployed static sensor tags using mobile devices carried by people (e.g., cell phones, handhelds, PDAs) that are equipped with RFID readers [9]. Compared to other alternative approaches for indoor sensing applications, this paradigm offers significant advantages in terms of cost and long-lived operation. This paradigm (Figure 1 illustrates the corresponding three-tier architecture), though similar to the data MULE approach [10, 11], allows for denser tag deployments in comparison. The underlying usage model also differs from other RFID-based sensing approaches such as in the WISP project [8].

We experimentally investigate the feasibility of RFID-based mobile sensor data gathering applications by focusing on UHF RFID devices and conducting a detailed characterization study in an indoor environment. We examine the impact of various factors, including reader mobility, multiple closely located tags and related key metrics. Our results show that read ranges around $1-2 \mathrm{~m}$ are feasible at walking speeds with careful planning, thus suggesting the feasibility of the considered class of applications. They also provide insights for optimizing read performance. For example, tag aggregation is proposed as an effective way to compensate for shorter read range.

Our work differs from the past experimental RFID read characterization studies $[12,13,14,15]$ in that we consider mobile reader 


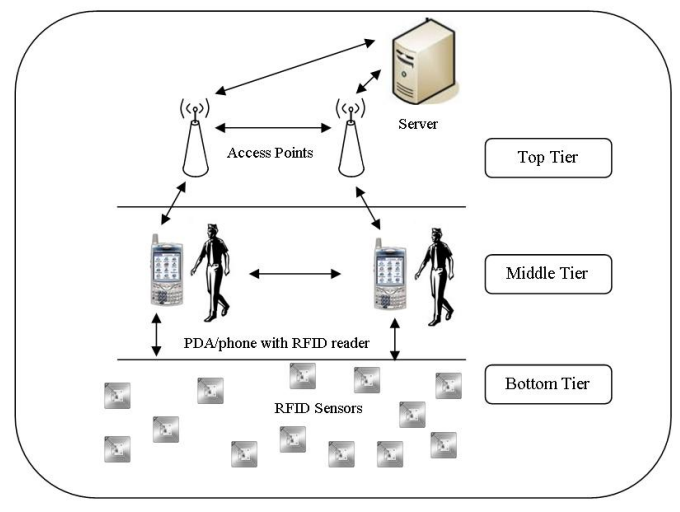

Figure 1: Three-tier architecture for RFID-based mobile sensor data gathering.

scenarios and look at reader-tag communication in the context of a specific class of applications, i.e., RFID-based mobile sensor data gathering applications.

\section{EXPERIMENTAL CHARACTERIZATION OF UHF RFID READ PROPERTIES IN INDOOR ENVIRONMENTS}

\subsection{Goals and Metrics}

In this section, we experimentally study read characteristics of state-of-the-art UHF RFID hardware in order to assess the feasibility of the RFID-based mobile sensor data gathering paradigm [9]. For this purpose, we evaluate the impact of a wide range of factors that could potentially affect read performance, including: relative reader-tag orientation, impact of multiple closely spaced tags and reader mobility. We use the following set of metrics to quantify read performance in our experiments:

- Read range, defined as the range at which at least $x \%$ of the read attempts are successful ( $x$ set to 10 in our experiments). This metric helps to conservatively estimate the read range by accounting for variations over time (due to multipath fading, for example).

- Reads per tag, defined as the average number of successful reads per tag in scenarios with multiple closely spaced tags.

Besides the above metrics, we also report measured read speed (i.e., reads per second) ${ }^{1}$ results from our experiments.

\subsection{Experiment Settings}

For determining the feasibility of RFID-based mobile sensor data gathering applications, we focus on reader-tag communication, the

\footnotetext{
${ }^{1}$ Note that read speed is referred to as read rate in [12].
}

core component of such applications, and carry out experimental characterization of read performance with the state-of-the-art RFID technology. Specifically, we consider RFID devices that communicate via far-field coupling and operate in the UHF bands (860$960 \mathrm{MHz}$ ) as they not only can provide larger read range, but also are more effective in supporting scenarios with multiple closely spaced tags as well as transfer of larger amounts of data.

The UHF hardware used in our characterization study is compliant with the EPC Class1 Gen2 standard [3]. We use A528 compact reader from Caen RFID [7], an Italian supplier of UHF hardware. This reader is based on the new Impinj Indy R1000 reader chip [16], which combines multiple components into an integrated RFID circuit, enabling digital signal processing and analog data processing on the same chip. The result is smaller reader size $(42 \times 60 \times 6.3$ $\mathrm{mm}^{3}$ ), making it suitable for use with handheld devices. Moreover, it complies with and can operate in both European (ETSI EN 302 208 ) and US (FCC part 15) regulatory environments. Although we have experimented with different antenna and tag types (see [9]), in this paper we only discuss results for one antenna type and one tag type due to space limitations. Specifically, we consider a $3 \mathrm{dBi}$ linear polarized Planar Inverted-F (PIFA) antenna for the reader. We use a simple UHF label tag from UPM Raflatac [17]; this tag is a printed circuit enclosed in thin flexible plastic with a sticky back.

In our experimental setup, the A528 reader mounted on A528DAT service board (providing power, USB and RS232 connections) is connected to a laptop over USB; it communicates with nearby tags via the antenna mentioned above. Note that we do not consider sensor or semi-passive tags, but it does not reduce the value of our study in assessing the feasibility of RFID-based mobile sensor data gathering applications because of two reasons. First, EPC Class1 Gen2 standard establishes a single UHF specification that can be easily extended to incorporate higher class tags while not conflicting with the operation of deployed tags. Note that semi-passive tags belong to class 3, whereas label tags used in our study belong to class 1 . Second, our study using passive tags in essence indicates worst-case read performance because higher class tags with built-in batteries typically have a larger read range because of their battery-assisted backscatter capability. We also do not consider the reader-AP communication as it is relatively less challenging; communication over $\mathrm{WiFi}$ in indoor scenarios with pedestrian mobility has been well studied with a number of performance optimizations.

Most of our experiments were carried out in a laboratory environment, a typical indoor scenario. The size of the lab is approximately $6.25 \mathrm{~m} \times 7 \mathrm{~m}$. It has side benches against the walls all the way round the room with several computers on them and a large table in the middle of the room; some of the benches have underneath them cupboards as well as two metal cabinets in one corner. We used two functions provided by the software supplied with the reader to calculate the metrics (see Section 3.1) for our experimental characterization: (1) single inventory, which attempts to read a tag in range around 60 times; and (2) start inventory, which continuously reads nearby tags until stopped.

\subsection{Results}

\subsubsection{Baseline Scenario and Impact of Tag Orienta- tion}

We begin our experimental characterization of UHF read properties by considering a baseline scenario. Specifically, we look at the communication between reader with $3 \mathrm{dBi}$ linear polarized PIFA antenna (which is the default antenna that came as part of A528 reader development kit) and a simple label tag in free space. In this scenario, the reader antenna is placed flat on the table in the middle 


\begin{tabular}{|l|c|}
\hline \hline Tag Orientation & Read Range $(\mathrm{cm})$ \\
\hline \hline Vertical & 200 \\
\hline Horizontal & 120 \\
\hline \hline
\end{tabular}

Table 1: Read range results for the baseline scenario - reader with 3dBi linear polarized PIFA antenna communicating with a label tag in free space.

of the lab and one tag is also placed on the same table such that it is standing either vertically or horizontally propped up from behind by a folded piece of paper also standing on the table. The separation distance between the reader antenna and the tag on the table is varied to determine the read range. Table 1 shows the results, which clearly show the impact of tag orientation on read range the read range when the tag is standing vertically and aligned with the electric field of the antenna is greater by more than $65 \%$ compared to the case the tag is standing horizontally. We also observed a steep fall effect - read success rate quickly drops to zero when the tag is moved further away from the reader antenna by a few centimeters.

\subsubsection{Multiple Tags in Close Proximity}

Results for the baseline scenario in the previous subsection suggest that read ranges in the order $1-2 \mathrm{~m}$ are feasible, but that may not be acceptable for some sensing applications. One straightforward approach to supporting such applications is to lower the spatial resolution of sensing by requiring multiple closely located tags together to provide a sample rather than every individual tag. While this can be done relatively inexpensively due to the lower cost of tags, it also increases the likelihood of collisions between responses from tags that are close to each other in turn reducing the effective read range. We have carried out experiments to assess the impact of tag proximity on read range. Our experimental setup is similar to the baseline scenario involving reader with PIFA antenna and label tags in free space, but now we have multiple (5) tags standing besides each other in a row; we vary the separation between adjacent tags from $0 \mathrm{~cm}$ to $30 \mathrm{~cm}$. As can be seen from Figure 2, $30 \mathrm{~cm}$ separation results in a range similar to the single tag scenario (see previous subsection), whereas no spacing between tags brings down the range by more than half. On the positive side, from the viewpoint of RFID-based mobile sensor data gathering applications, $30 \mathrm{~cm}$ inter-tag spacing is low enough that it would be acceptable for most applications.

We have also considered the impact of number of tags and found that increasing the number of tags for a given inter-tag spacing has the effect of reducing the read range. For instance, we found the read range with 10 tags and $5 \mathrm{~cm}$ spacing between adjacent tags to be $88 \mathrm{~cm}$, compared to $127 \mathrm{~cm}$ with 5 tags and $5 \mathrm{~cm}$ spacing shown in Figure 2. We also experimented with tags placed in a grid-like manner. The results from these experiments are qualitatively similar to the above where tags are placed in a row (linear arrangement). Another important observation from these experiments is that the read speed measured in our setting is around 150 reads/second, about one-third of the maximum read speed (450 reads/second) possible with the EPC Class 1 Gen 2 standard.

\subsubsection{Mobility}

In this subsection, we investigate a key issue for enabling RFIDbased mobile sensor data gathering applications - the ability to read stationary tags from a distance using mobile readers carried by people moving at typical walking speeds $(1 \mathrm{~m} / \mathrm{s})$ in indoor sce-

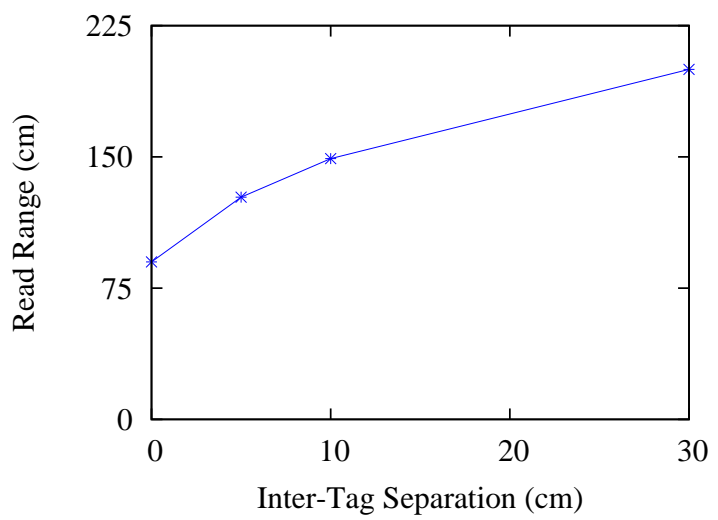

Figure 2: Impact of multiple closely spaced tags on read range. In this scenario, reader with $3 \mathrm{dBi}$ linear polarized PIFA antenna is communicating with 5 vertically standing label tags separated from each other by different distances.

narios. In our experimental setup, 5 label tags are placed vertically $30 \mathrm{~cm}$ apart (based on the results from the last subsection) and the bottom end of each tag is stuck to the side bench such that much of the tag is visible from all sides (as if the tag was in free space). The experiment involved a person carrying the reader with $3 \mathrm{dBi}$ linear PIFA antenna walking on a line parallel to the side bench and separated by a distance $d$. We experimented with different values of $d$. A start inventory operation is begun at one end of the walk and it is stopped at a point where none of the tags could be read; this took around 2-3 single inventory cycles. Figure 3 shows the average number of reads per tag obtained from our measurements for distances that ensured at least 5 reads per each tag; each data point in the figure is an average over all 5 tags and 10 different walks. As expected, the number of reads per tag drops considerably as the distance $d$ is increased. We also observed that the number of reads for different tags varies widely as $d$ is increased. But more importantly, the tags can be reliably read while walking from a distance of up to $180 \mathrm{~cm}$, which is only $10 \%$ lower than the read range in a scenario with stationary reader and single tag (see results from baseline scenario). This is an encouraging result for RFID-based mobile sensor data gathering. In order to look at the impact of speed, we carried out some experiments with slower (faster) walking and found that it increases (reduces) readable distance compared to normal walking experiment as one would expect.

Though not discussed in this paper, we have also conducted several other experiments to study the impact of presence of people, reader antenna and tag types, different types of obstacles (e.g., plasterboard wall, brick wall), material surfaces (e.g., wood, metal) and height difference between readers and tags [9].

\section{CONCLUSIONS}

In this paper, we have looked into the feasibility of indoor RFIDbased mobile sensor data gathering applications via a detailed experimental characterization study involving UHF RFID devices. Our results show that it is possible to obtain read range between $1-2 \mathrm{~m}$ at walking speeds with current technology through careful planning, especially in terms of relative orientation of reader antennas and tags. Aggregating multiple tags into a single high-level tag from the application viewpoint has the dual benefit of compensating for shorter read ranges as well as dealing with tag disorientation. Overall, from the viewpoint of RFID-based mobile sensor 


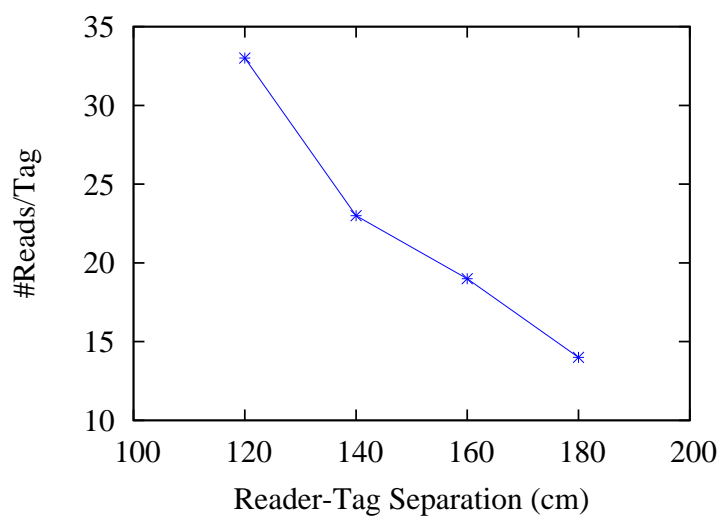

Figure 3: Read performance with a mobile reader with $3 \mathrm{dBi}$ linear PIFA antenna and label tags placed vertically in free space on a line parallel to the reader's path at walking speeds.

data gathering, our results show feasibility of such applications as seen from the observed read ranges relative to typical width of corridors $(2 \mathrm{~m})$. Our future work will focus on multi-reader scenarios and studying the behavior of end-to-end latency and data delivery reliability metrics.

\section{REFERENCES}

[1] K. Finkenzeller. RFID Handbook. Wiley, second edition, 2003.

[2] R. Want. An Introduction to RFID Technology. IEEE Pervasive Computing, 5(1):25-33, Jan 2006.

[3] EPCglobal Specification for RFID Air Interface. EPC Radio-Frequency Identity Protocols Class-1 Generation-2 UHF RFID Protocol for Communications at $860 \mathrm{MHz}-960$ $\mathrm{MHz}$ Version 1.1.0, Dec 2005.

[4] R. Want. Enabling Ubiquitous Sensing with RFID. IEEE Computer, 37(4):84-86, Apr 2004.

[5] H. Liu et al. Integration of RFID and Wireless Sensor Networks. In Proc. 5th ACM SenSys Workshops, 2007.

[6] KSW-Microtec. http://www.ksw-microtec.de/.

[7] CAEN RFID. http://www.caen.it/rfid/.

[8] M. Philipose et al. Battery-Free Wireless Identification and Sensing. IEEE Pervasive Computing, 4(1):37-45, Jan 2005.

[9] I. A. Currie and M. K. Marina. A Case for RFID-based Mobile Sensor Data Gathering in Indoor Environments. The University of Edinburgh, Informatics Research Report EDI-INF-RR-1291, Oct 2008

http://homepages.inf.ed.ac.uk/mmarina/papers/EDI-INF-RR1291.pdf.

[10] R. C. Shah, S. Roy, S. Jain, and W. Brunette. Data MULEs: Modeling and Analysis of a Three-Tier Architecture for Sparse Sensor Networks. Elsevier Ad Hoc Networks, 1(2-3): 215-233, Sep 2003.

[11] S. Jain et al. Exploiting Mobility for Energy Efficient Data Collection in Wireless Sensor Networks. Springer Mobile Networks and Applications (MONET), 11(3):327-339, Jun 2006.

[12] K. M. Ramakrishnan and D. D. Deavours. Performance Benchmarks for Passive UHF RFID Tags. In Proc. 13th GI/ITG Conference on Measurement, Modeling, and Evaluation of Computer and Communication Systems, 2006.
[13] S. R. Aroor and D. D. Deavours. Evaluation of the State of Passive UHF RFID: An Experimental Approach. IEEE Systems Journal, 1(2):168-176, Dec 2007.

[14] S. Hodges, A. Thorne, H. Mallinson, and C. Floerkemeier. Assessing and Optimizing the Range of UHF RFID to Enable Real-World Pervasive Computing Applications. In Proc. 5th International Conference on Pervasive Computing (Pervasive'07), 2007.

[15] M. Buettner and D. Wetherall. An Empirical Study of UHF RFID Performance. In Proc. ACM MobiCom, 2008.

[16] Impinj Indy R1000 UHF RFID Reader Chip. http://www.impinj.com/.

[17] UPM Raflatac. http://www.upmraflatac.com/. 